\title{
Multivariate Model for Test Response Analysis
}

\author{
Shaji Krishnan \\ Analytical Research Department \\ TNO \\ Zeist, The Netherlands \\ Email: shaji.krishnan@tno.nl
}

\author{
Hans G. Kerkhoff \\ CTIT-TDT \\ University of Twente \\ Enschede, The Netherlands \\ Email: h.g.kerkhoff@utwente.nl
}

\begin{abstract}
A systematic approach to construct an effective multivariate test response model for capturing manufacturing defects in electronic products is described. The effectiveness of the model is demonstrated by its capability in reducing the number of test-points, while achieving the maximal coverage attainable by the specific test method on an industrial circuit.
\end{abstract}

\section{INTRODUCTION}

Since the parametric variation and the measurement bias have become integral part of VLSI manufacturing and test environment, the paradigm of test response analysis has shifted from univariate [1] to multivariate analysis [2]. In a univariate analysis, only one test response variable is analyzed at a time. In contrast, in a multivariate analysis, a third artificial variable, which is a combination of at least two test response variables, is analyzed via a regression scheme. To circumvent certain limitations of the regression schemes methods, like the choice of test variable to the amount of correlation among test variables, this paper proposes an alternative multivariate test response model based on the Principal Component Analysis (PCA) and Mahalanobis Distance (MD).

\section{PCA-MD based Multivariate Response Model}

The PCA-MD based Multivariate Response Model, capable of classifying devices into non-defective or defective devices, is based on the Principal Component Analysis procedure for variable reduction [3], in combination with the multivariate distance metric the Mahalanobis distance [4]. The model is parameterized by a set of measurement variables that remain significant for such a classification. Once such a parameter variable set has been designated, a reliable metric is computed from the set of measurements corresponding to the significant variables. The empirical distribution of the metric so determined from $k$ samples of the golden devices is utilized to classify the arbitrary device into non-defective or defective device.

\section{EXPERIMENTS AND RESULTS}

A full analogue RF product is used to demonstrate the applicability of the PCA-MD based multivariate response model for classifying devices into non-defective and defective ones. Table I shows the results of 3 multivariate analysis techniques: the PCA-MD (PCA-MD based multivariate response analysis technique), VRT [5] and the PCA [6], applied to the measurement responses of Known Defective Devices (KDDs) to the supply ramp test. For each technique, the \#Testpoints indicate the number of response variables chosen, while the Testpoint indicates the index of the response variable. The $\%$ $\mathrm{R}$. Coverage is the percentage relative to the coverage of the PCA-MD based multivariate response analysis technique.

TABLE I

COMPARATIVE RESUlTS

\begin{tabular}{llll}
\hline Method & \#Testpoints & Testpoint & \% R. Coverage \\
\hline \hline PCA-MD & 2 & 8,14 & 100 \\
VRT & 5 & $\{19,20\},\{20,21\},\{16,17\}$ & 99.04 \\
PCA & 24 & $\{1,2, . .24\}$ & 96.21 \\
\hline
\end{tabular}

The relative coverage of a PCA technique with all the testpoint measurements was less compared to the PCA-MD and VRT techniques. The number of test points for the PCA-MD was lower compared to the PCA and VRT and yielded the maximum coverage.

\section{Conclusions}

We have described a multivariate test response modeling technique based on a PCA variable reduction technique and Mahalanobis distance metric. The advantage of this modeling technique is that it chooses only the most relevant measurement data for classification purposes. The requirement for a high amount of positive correlation among pair of measurement variable, imposed by the VRT technique, was relaxed by choosing a suitable metric in the proposed model. The invariance of the metric to scaling extends the flexibility of the applying the model to heterogeneous production environments.

\section{REFERENCES}

[1] M. Sachdev, P. Janssen, and V. Zieren, "Defect detection with transient current testing and its potential for deep sub-micron cmos ics," in Proc. IEEE International Test Conference (ITC), 1998, pp. $204-213$.

[2] L. Fang, M. Lemnawar, and Y. Xing, "Cost effective outliers screening with moving limits and correlation testing for analogue ics," in Proc. IEEE International Test Conference (ITC), 2006.

[3] T. Jollifee, Principal Component Analysis. Springer-Verlag, New York, 2002.

[4] P. C. Mahalanobis, "On the generalised distance in statistics," in Proc. of the National Institute of Sciences of India, vol. 2, no. 1, 1936, pp. 49 55.

[5] S. Krishnan, R. Jonker, and L. van de Logt, "Variance reduction for supply ramp based cheap rf test alternatives," in IEEE European Test Symposium, 2007, pp. $55-62$.

[6] S. Krishnan, "Signature analysis for enhancing resolution of analogue test," in IEEE International Design and Test Workshop, 2006. 